\title{
What's New and Plans for the Future
}

The journal is pleased to publish the abstracts of the $7^{\text {th }}$ European Conference of Infections in Obstetrics and Gynecology that was hosted by the European Society for Infectious Diseases in Obstetrics and Gynecology (EISDOG) October 4-6 in Istanbul, Turkey. The addition of ESIDOG to the annual publication of abstracts from the meetings sponsored by IDSOG and I-IDSOG USA permits the journal to serve as a common resource for dissemination of information regarding infectious diseases in women worldwide. Thus, the journal is becoming a repository of information from around the world. It has grown significantly since its inception in 1994 and is now the official publication of the Infectious Disease Society for Obstetrics and Gynecology and the International Infectious Disease Society for Obstetrics and Gynecology - USA.

The future of the journal is bright, and plans are currently underway to expand its content. The latest addition is a case review that uses the basic principles of microbiology and infectious diseases as they apply to the diagnosis and management of specific clinical cases. The journal encourages our readers to submit interesting cases that can serve to teach others for publication in the Case Review section.

The journal also encourages the submission of letters and questions to the editor that comment on published articles and protocols, or raises issues of common and general interest. This journal is a vehicle for physicians and researchers with an interest in infectious diseases, specifically of women. Through this publication, information on women's health with regard to infectious disease can be maintained on a current level and disseminated worldwide.

Sebastian Faro, MD, PhD 


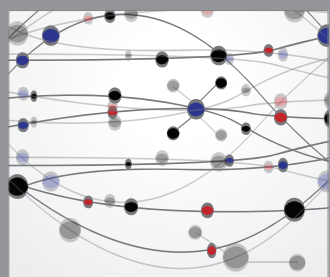

The Scientific World Journal
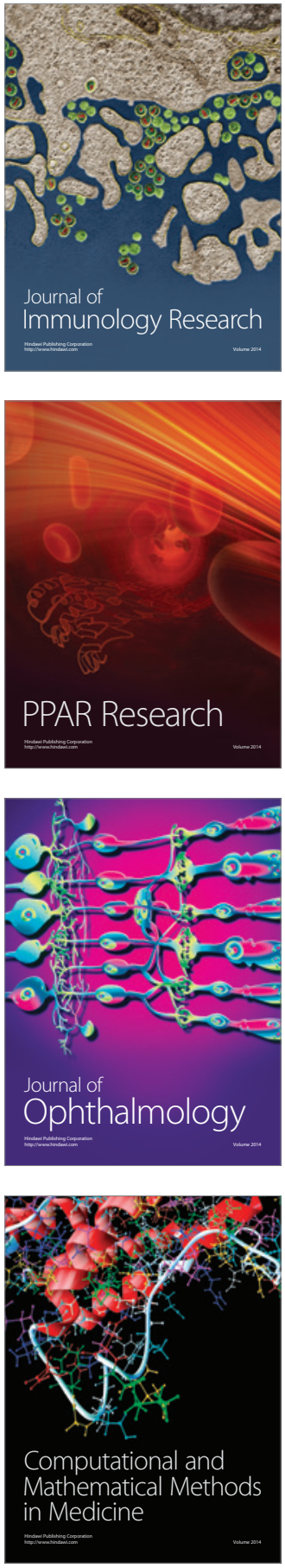

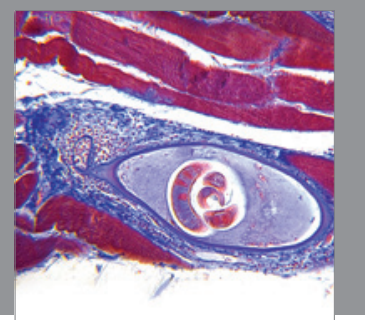

Gastroenterology

Research and Practice
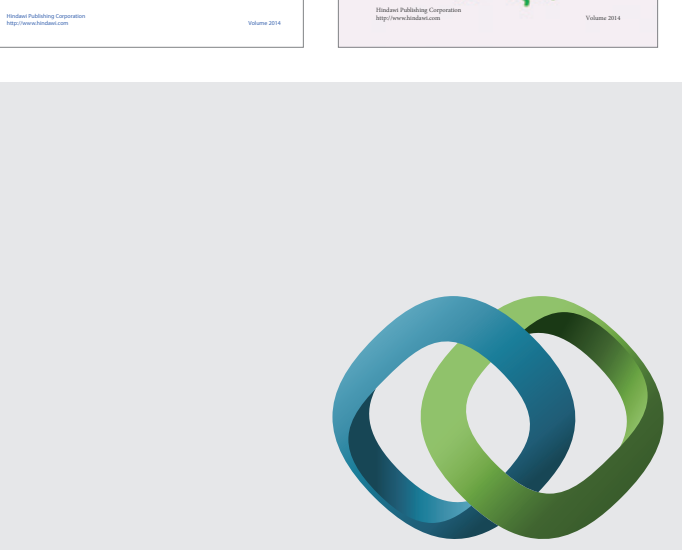

\section{Hindawi}

Submit your manuscripts at

http://www.hindawi.com
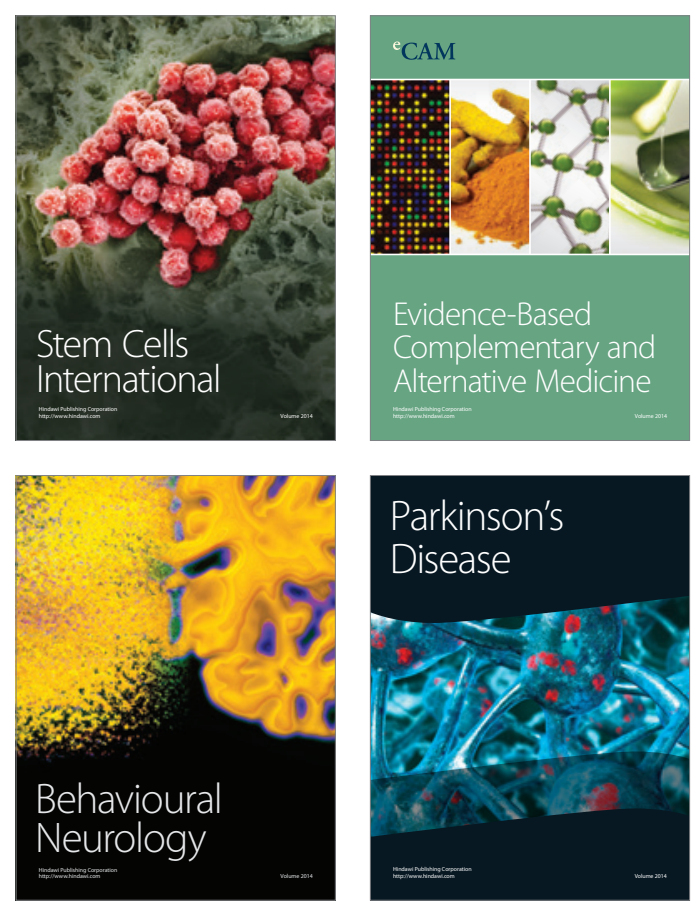

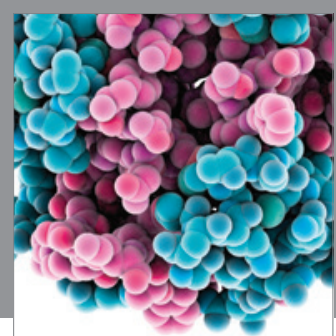

Journal of
Diabetes Research

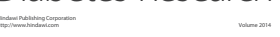

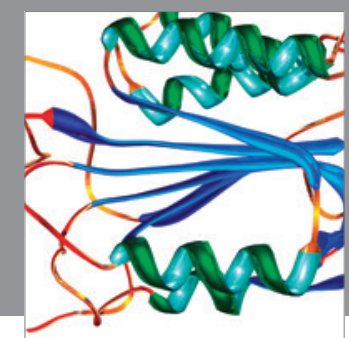

Disease Markers
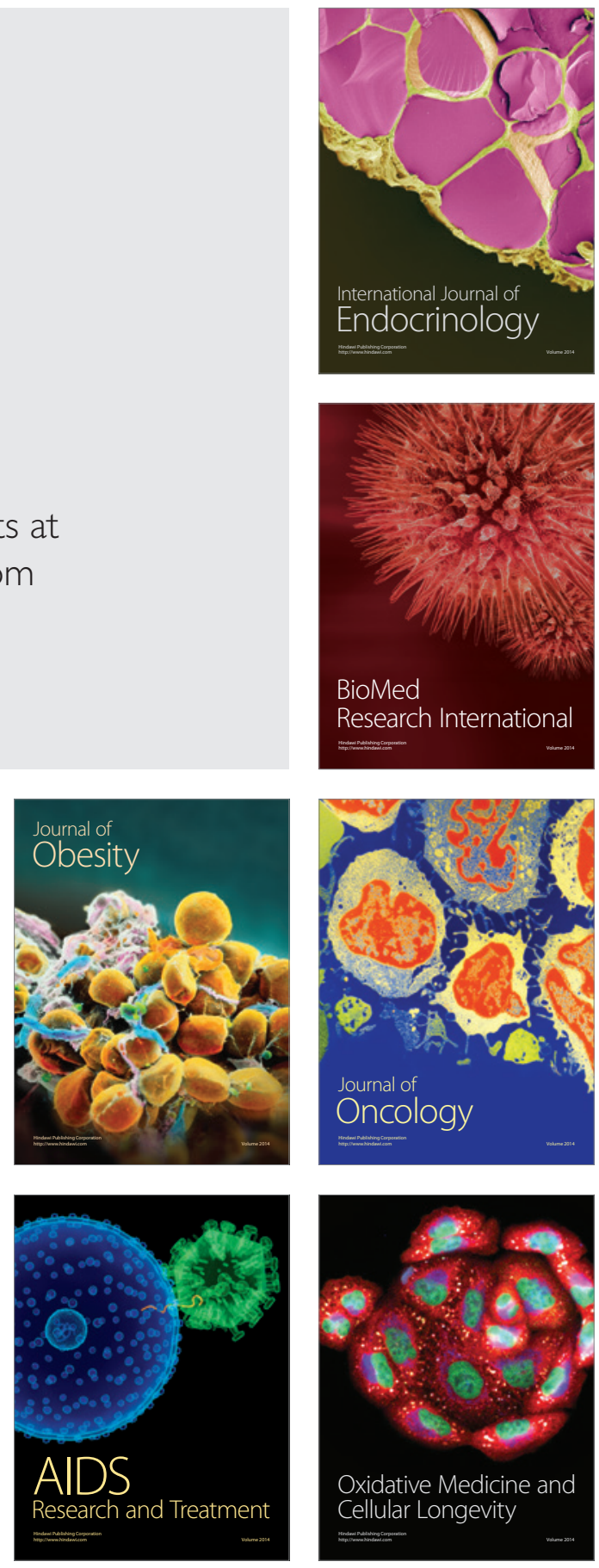